\title{
OPEN Autoregulation and dual stepping mode of MYA2, an Arabidopsis myosin XI responsible for cytoplasmic streaming
}

\author{
Takeshi Haraguchi ${ }^{1}$, Kohji Ito ${ }^{1 凶}$, Takamitsu Morikawa ${ }^{2}$, Kohei Yoshimura ${ }^{1}$, Nao Shoji ${ }^{1}$, \\ Atsushi Kimura ${ }^{1}$, Mitsuhiro Iwaki ${ }^{2 \bowtie}$ \& Motoki Tominaga ${ }^{3,4 凶}$
}

Arabidopsis thaliana has 13 genes belonging to the myosin XI family. Myosin XI-2 (MYA2) plays a major role in the generation of cytoplasmic streaming in Arabidopsis cells. In this study, we investigated the molecular properties of MYA2 expressed by the baculovirus transfer system. Actin-activated ATPase activity and in vitro motility assays revealed that activity of MYA2 was regulated by the globular tail domain (GTD). When the GTD is not bound to the cargo, the GTD inhibits ADP dissociation from the motor domain. Optical nanometry of single MYA2 molecules, combining total internal reflection fluorescence microscopy (TIRFM) and the fluorescence imaging with one-nanometer accuracy (FIONA) method, revealed that the MYA2 processively moved on actin with three different step sizes: $-28 \mathrm{~nm}$, $29 \mathrm{~nm}$, and $60 \mathrm{~nm}$, at low ATP concentrations. This result indicates that MYA2 uses two different stepping modes; hand-over-hand and inchworm-like. Force measurement using optical trapping showed the stall force of MYA2 was $0.85 \mathrm{pN}$, which was less than half that of myosin V (2-3 pN). These results indicated that MYA2 has different transport properties from that of the myosin $\mathrm{V}$ responsible for vesicle transport in animal cells. Such properties may enable multiple myosin XIs to transport organelles quickly and smoothly, for the generation of cytoplasmic streaming in plant cells.

Myosin is a motor protein that converts the chemical energy liberated by ATP hydrolysis into directed movement along actin filaments. Phylogenetic analyses of myosin sequences have shown that there are at least 79 myosin classes in eukaryotes ${ }^{1}$. The myosin superfamily shares a common domain composition: a conserved motor domain (MD) with ATPase and actin-binding activities, a neck domain comprising one to six repeats of isoleucine-glutamine (IQ) motifs acting as a lever arm, a coiled-coil domain, and a globular tail domain (GTD) that binds the cargo ${ }^{2}$. Many myosins of different classes participate in transport of various cargos, such as organelles, vesicles, and ribonucleoproteins, within cells ${ }^{3}$. Myosins bind different cargos via the GTD and transport them along actin filaments by the MD activity. Proper delivery and regulation of intracellular transport by myosins within cells is crucial for maintaining cellular function. The myosin motility is finely regulated by various mechanisms such as phosphorylation, calcium ions, and autoinhibition ${ }^{4,5}$. It has been reported that the activity of myosin V, myosin VIIA, and myosin X is regulated by autoinhibition using the GTD. In these myosins, the MD is typically maintained in an OFF state when the GTD is folded over and interacts with the MD to inhibit ATPase activity. Regulation by autoinhibition could avoid unproductive movements on actin filaments without cargo and wasteful ATP consumption. Conserved amino acid residues on both the MD and the GTD of myosin $\mathrm{V}$ mediate this interaction ${ }^{6-8}$.

Cytoplasmic streaming is the long-range, rotational movement of cytoplasm, widely observed in cells of organisms ranging from algae to angiosperms ${ }^{9}$. Cytoplasmic streaming is generated by organelle-associated plant-specific class XI myosin sliding along actin filaments ${ }^{10}$. Myosin XI has a typical myosin structure, consisting of an $\mathrm{N}$-terminal MD, a neck domain comprising six tandem repeats of IQ motifs, a long coiled-coil domain for dimerization, and a C-terminal GTD that binds cargo. The molecular mechanism of myosin XI that generates fast cytoplasmic streaming has been investigated using tobacco $175 \mathrm{kDa}$ mosin XI purified from cultured

\footnotetext{
${ }^{1}$ Department of Biology, Graduate School of Science, Chiba University, Chiba 263-8522, Japan. ${ }^{2}$ RIKEN Center for Biosystems Dynamics Research, RIKEN, Osaka, Japan. ${ }^{3}$ Faculty of Education and Integrated Arts and Sciences, Waseda University, 2-2 Wakamatsu-cho, Shinjuku-ku, Tokyo 162-8480, Japan. ${ }^{4}$ Major in Integrative Bioscience and Biomedical Engineering, Graduate School of Science and Engineering, Waseda University, 2-2 Wakamatsu-cho, Shinjuku-ku, Tokyo 162-8480, Japan. ${ }^{\boxplus}$ email: k-ito@faculty.chiba-u.jp; iwaki@riken.jp; motominaga@waseda.jp
} 
a

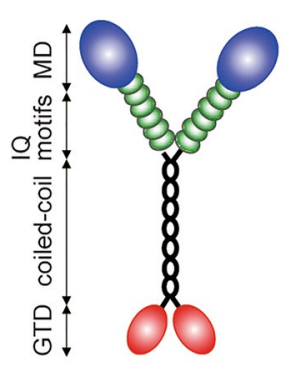

Full b
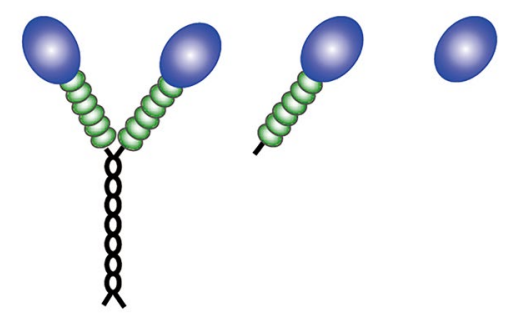

HMM

$6 I Q$

MD

GTD

Figure 1. Schematic diagrams of the MYA2 constructs used in this work. (a) Full: Full-length construct comprising MD, six IQ motifs to which six calmodulins bind, coiled-coil, and GTD domains. (b) HMM: HMM construct comprising of MD, six IQ motifs to which six calmodulins bind, and a coiled-coil domain. 6IQ: 6IQ construct comprising of MD and six IQ motifs to which six calmodulins bind. MD: MD construct comprising only MD. GTD: GTD construct comprising only GTD.

tobacco BY-2 cells. Tobacco $175 \mathrm{kDa}$ myosin XI moves processively along actin filaments with approximately $35 \mathrm{~nm}$ steps ${ }^{11}$. The processive stepping motion is analogous to that of myosin Va, which is involved in intracellular transport in mammalian cells and moves on actin filaments in a "hand-over-hand" fashion ${ }^{2}$. Although the structural and motile features of tobacco $175 \mathrm{kDa}$ myosin XI were similar to those of myosin Va, the $V_{\max }$ value of the velocity and of the ATPase activity of tobacco $175 \mathrm{kDa}$ myosin XI were $4.6 \mu \mathrm{m} \mathrm{s}^{-1}$ and $76 \mathrm{~s}^{-1}$, respectively, which are about tenfold higher than those of myosin $\mathrm{Va}^{11}$.

Genome analysis revealed that Arabidopsis thaliana possesses 13 myosin XI members ${ }^{12}$. Gene knockout studies have revealed that myosin XI-1, XI-2, XI-B, XI-I, and XI-K are responsible for the movement of organelles, such as the endoplasmic reticulum, Golgi stacks, peroxisomes, and mitochondria ${ }^{13-17}$, concomitant with growth defects ${ }^{15,16,18}$. The full-length cDNAs and promoter regions for all 13 Arabidopsis myosin XIs have been cloned, and their tissue-specific expression and motile and enzymatic activities were identified ${ }^{19}$. The velocities and ATPase activities of the 13 Arabidopsis myosin XIs are significantly different, and the myosins are classified broadly into three groups-high, medium, and low-based on velocity. The velocity grouping appears to be roughly correlated with the tissue-specific expression patterns ${ }^{19}$. Gene knockout analysis indicated that Arabidopsis myosin XI-2 (MYA2) and XI-K are the major motor proteins that provide the motive force for cytoplasmic streaming ${ }^{16,17}$. Among 13 Arabidopsis myosin XIs, MYA2 is the closest to tobacco $175 \mathrm{kDa}$ myosin XI phylogenetically. It is unclear whether the motile mechanisms of MYA2 is the same as that of tobacco $175 \mathrm{kDa}$ myosin XI. In this study, the in vitro enzymatic properties and single-molecule motility of recombinant Arabidopsis MYA2 were analyzed. We found that MYA2 has different motile and regulatory properties than those of tobacco $175 \mathrm{kDa}$ myosin XI.

\section{Results}

Constructs. A schematic diagram of full-length MYA2 (Full) deduced from its amino acid sequence is shown in Fig. 1a. In general, the domain presence and composition were consistent across all 13 Arabidopsis myosin XIs ${ }^{20}$. Arabidopsis myosin XI is composed of a MD, a neck domain with six IQ motifs to which calmodulin or calmodulin-like proteins bind, a coiled-coil region, and a GTD (Fig. 1a) ${ }^{9,19,20}$. In this study, we generated five types of recombinant MYA2 constructs: Full (Fig. 1a); heavy meromyosin which lacked GTD (HMM); motor domain with six IQ motifs (6IQ); MD; and GTD (Fig. 1b) ${ }^{19}$. These constructs were expressed using a baculovirus transfer system in High Five insect cells. The recombinant MYA2 proteins were purified using both nickel-affinity and Flag-affinity resin, as previously reported ${ }^{19,21}$. SDS-PAGE analysis was performed to confirm the purity and homogeneity of the proteins (Fig. S1a), and binding of calmodulins to the purified 6IQ (Fig. S1b).

Inhibitory effects of GTD on actin-activated ATPase activities. Figure 2a shows the actin-activated ATPase activities of MYA2 constructs at various actin concentrations. Plots of ATPase activity against actin concentration were found to fit a Michaelis-Menten type curve. Using this curve, we determined the maximum rate of ATP turnover $\left(V_{\max }\right)$ and the actin concentration at which the ATPase rate reached half of its maximum $\left(K_{\mathrm{m}}\right)$. The $V_{\max }$ values of HMM, 6IQ, and MD were $99 \mathrm{~s}^{-1}, 127 \mathrm{~s}^{-1}$, and $56 \mathrm{~s}^{-119}$, respectively (Fig. 2a). In contrast, the $V_{\max }$ value of Full was $8.1 \mathrm{~s}^{-1}$ much smaller than those of HMM, 6IQ, and MD (Fig. 2a). The low $V_{\max }$ value of Full was likely due to the inhibitory effect of the GTD on actin-activated ATPase activity. To confirm inhibition by the GTD, the actin-activated ATPase activities of HMM, 6IQ, and MD were measured in the presence of various concentrations of GTD. The GTD inhibited the actin-activated ATPase activities of HMM and 6IQ in a concentration-dependent manner, but not that of MD (Fig. 2b).

The actin-activated ATPase activities of HMM and 6IQ in the presence of various concentrations of GTD and $23.8 \mu \mathrm{M}$ actin were fit to a hyperbola, defining an inhibition constant $\left(K_{\mathrm{i}}\right)$ of GTD. The $K_{\mathrm{i}}$ values of GTD for HMM and 6IQ were $0.5 \mu \mathrm{M}$ and $0.8 \mu \mathrm{M}$, respectively (Fig. 2b). These results suggest that the GTD inhibits the actin-activated ATPase activities of MYA2, and that this inhibition requires IQ motifs. Figure $2 \mathrm{c}$ shows the 
a

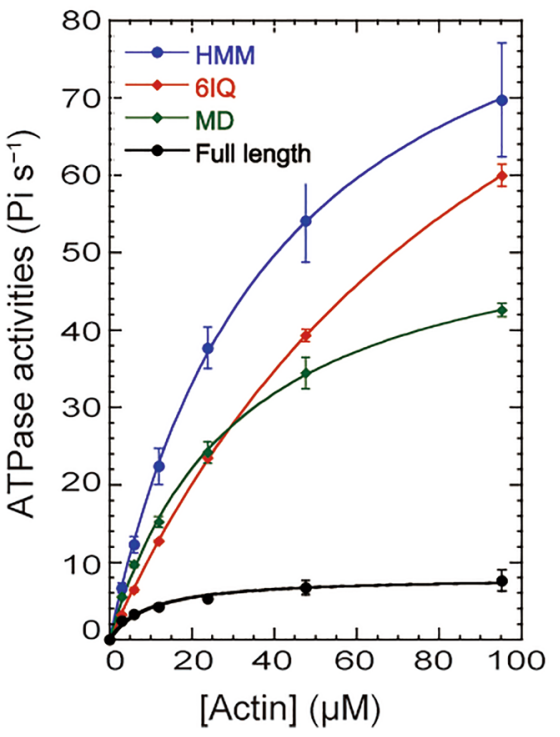

b

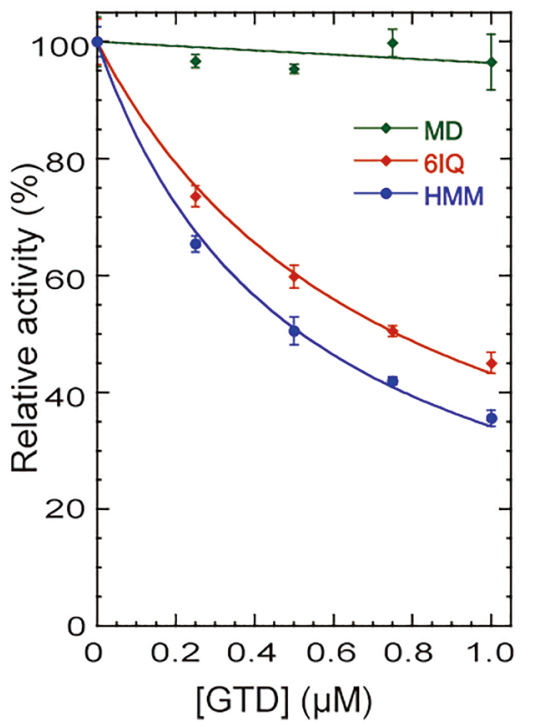

C

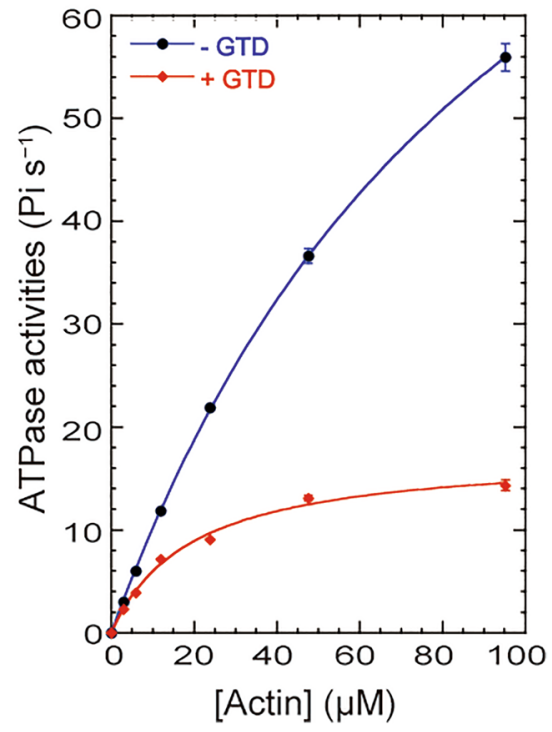

Figure 2. Actin-activated $\mathrm{Mg}^{2+}$-ATPase activities and inhibitory effects on $\mathrm{Mg}^{2+}$-ATPase activities of Full, HMM, 6IQ, and MD. (a) Actin-activated $\mathrm{Mg}^{2+}$-ATPase activities of Full, HMM, 6IQ, and MD. $\mathrm{Mg}^{2+}$-ATPase activities in the presence of various actin concentrations. The data were fitted using Michaelis-Menten dynamics, and the $V_{\max }$ and $\mathrm{K}_{\mathrm{app}}$ values were measured. The concentrations of Full, HMM, 6IQ, and MD used $0.005-0.019,0.001-0.009,0.002-0.018$, and 0.001-0.008 $\mu \mathrm{M}$, respectively. (b) Inhibitory effects of GTD on $\mathrm{Mg}^{2+}$-ATPase activities of HMM, MD, and 6IQ. The actin-activated ATPase activities of HMM and 6IQ in the presence of various concentrations of GTD and $23.8 \mu \mathrm{M}$ actin were fit to a hyperbola, and the $K_{\mathrm{i}}$ values of GTD for HMM and 6IQ were calculated to be $0.5 \mu \mathrm{M}$ and $0.8 \mu \mathrm{M}$, respectively. (c) Inhibitory effects of $\mathrm{Mg}^{2+}$-ATPase activities of 6IQ by $1 \mu \mathrm{M}$ GTD in the presence of various actin concentrations.

actin-activated ATPase activities of 6IQ in the presence of $1 \mu \mathrm{M}$ GTD and the absence of GTD. The $V_{\max }$ values of 6IQ in the absence and presence of GTD were $127 \mathrm{~s}^{-1}$ and $18 \mathrm{~s}^{-1}$, respectively. The $K_{\mathrm{m}}$ values of 6IQ for actin in the presence of $1 \mu \mathrm{M}$ GTD and in the absence of GTD were $19 \mu \mathrm{M}$ and $106 \mu \mathrm{M}$, respectively. At all actin concentrations, ATPase activities in the absence of the GTD were higher than ATPase activities in the presence of the GTD. The $V_{\max }$ value decreased by about seven times in the presence of $1 \mu \mathrm{M}$ GTD.

A similar inhibitory effect of GTD on the actin-activated ATPase activity has been reported for myosin V. Electron microscopy studies have shown that in the absence of cargo, full-length myosin V takes a folded-structure, in which the GTD bound to the head inhibits the actin-activated ATPase activity ${ }^{22}$. Because the inhibitory effects of GTD on the actin-activated ATPase activity and the myosin structure were similar between myosin $\mathrm{V}$ and MYA2, as shown above, the mechanism of inhibition by the GTD may be similar for the two myosins (Fig. 3c).

The inhibitory effect of GTD on the ATPase activity of full-length myosin V is released by $\mathrm{Ca}^{2+23}$. To investigate whether the $\mathrm{Ca}^{2+}$ releases the GTD-induced inhibitory effect of full-length MYA2, similar to that of myosin $\mathrm{V}$, we measured the actin-activated $\mathrm{Mg}^{2+}$ ATPase activity of Full in the presence of $\mathrm{Ca}^{2+}$. The actin-activated ATPase activities of Full did not increase but decreased by $30 \%$ by adding $100 \mu \mathrm{M} \mathrm{Ca}^{2+}$ (Fig. S2a). This result shows that, unlike in the case of myosin $\mathrm{Va}, \mathrm{Ca}^{2+}$ did not release the GTD-induced auto-inhibitory state of MYA2. The decrease in the activities by $\mathrm{Ca}^{2+}$ may be due to the dissociation of some of the calmodulin from IQ motifs.

Inhibitory effects of GTD on motility and ADP dissociation from acto-myosin. To investigate the inhibitory effect of GTD on the MYA2 motility in an in vitro motility assay, we measured the actin sliding velocity by 6IQ in the presence of exogenous free GTD because the C-terminus of Full interacts with the glass surface, thus inhibiting the binding of the Full GTD to MD.

6IQ moved actin filaments at $5.2 \mu \mathrm{m} \mathrm{s}^{-1}$ in the presence of $3 \mathrm{mM}$ ATP, which was similar to the value of Full ${ }^{19}$. In the presence of $1 \mu \mathrm{M}$ GTD, the velocity dropped to less than half of that in the absence of the GTD (Fig. 3a). This result indicates that GTD inhibited the motility of MYA2 containing IQ motifs, as well as the actin-activated $\mathrm{Mg}^{2+}$ ATPase activity. For most myosins, including myosin XI, the actin sliding velocity is mainly determined by the ADP dissociation rate from acto-myosin ${ }^{24-27}$. The ADP dissociation rate from acto-6IQ in the presence and absence of the GTD was determined by measuring the decrease in the fluorescence intensity of mant-ADP using the stopped-flow apparatus ${ }^{24-29}$ as described in "Methods". In the absence of GTD, the ADP dissociation rate from acto-6IQ was $430 \pm 40 \mathrm{~s}^{-1}$. In the presence of $1 \mu \mathrm{M} \mathrm{GTD}$, the rate was $22 \pm 3.4 \mathrm{~s}^{-1}$, which is about $1 / 20$ of that in the absence of GTD (Fig. 3b). These results suggest that free GTD, not bound to the cargo, inhibits the dissociation of ADP from MYA2 containing IQ motifs (Fig. 3c).

Then, we investigated whether $\mathrm{Ca}^{2+}$ releases the inhibitory effect of GTD on HMM motility. The velocity of HMM in the presence of $1 \mu \mathrm{M}$ GTD did not increase but decreased by $30 \%$ by adding $100 \mu \mathrm{M} \mathrm{Ca}^{2+}$ (Fig. S2b). 
a

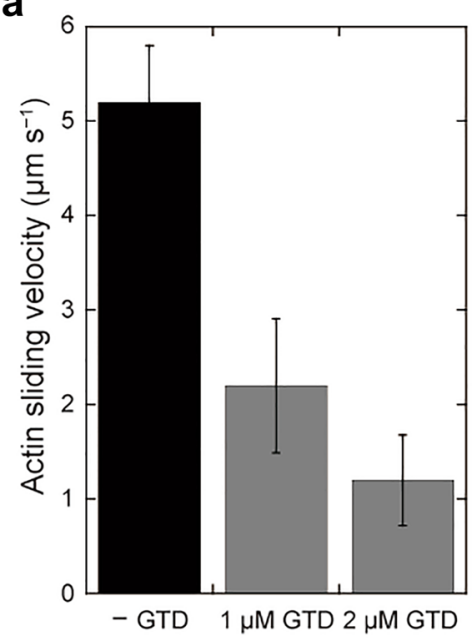

C

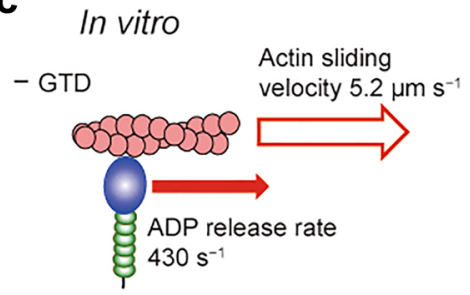

In vivo

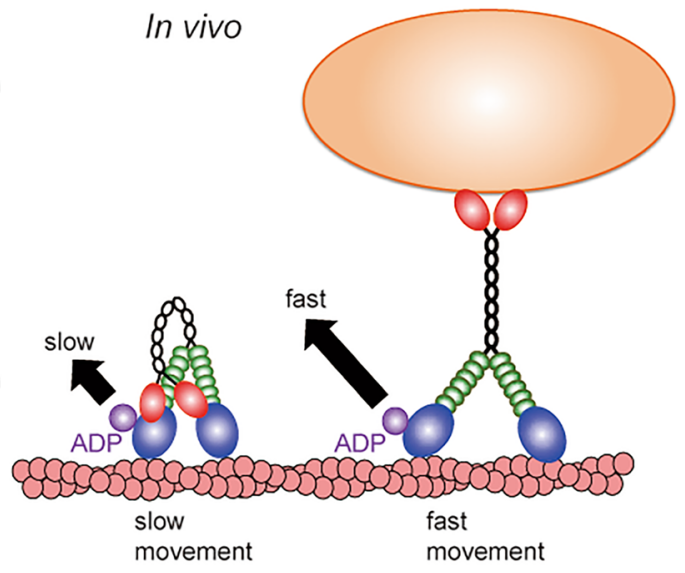

b
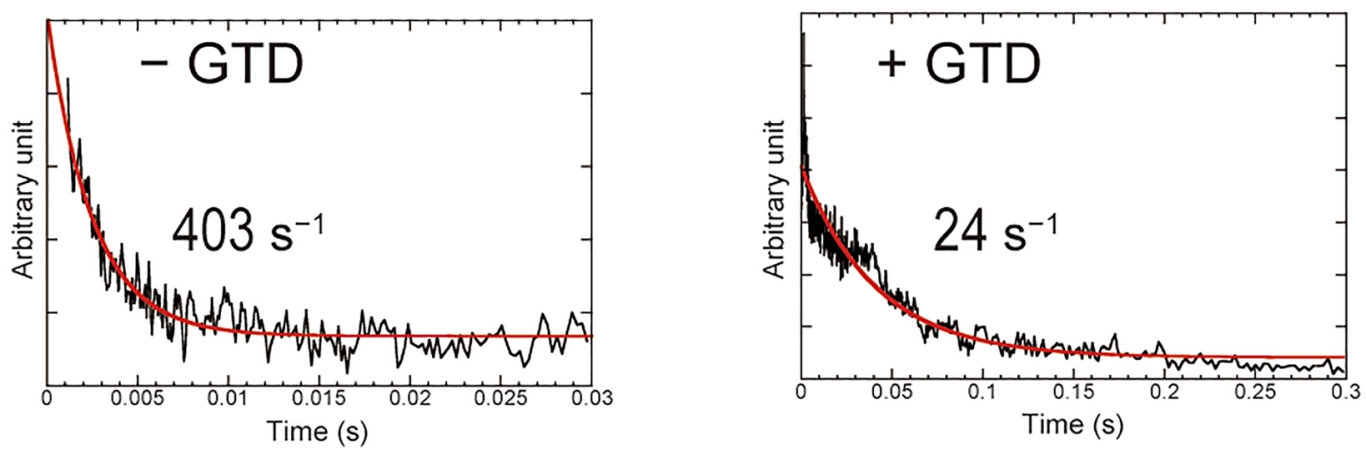

Figure 3. Inhibitory effects of actin sliding velocities of 6IQ and the dissociation rates of mant-ADP from 6IQ. (a) Inhibitory effects of actin sliding velocities of 6IQ by $1 \mu \mathrm{M}$ GTD and $2 \mu \mathrm{M}$ GTD in the presence of $3 \mathrm{mM}$ ATP. (b) Mant-ADP dissociation from acto-6IQ in the presence of $1 \mu \mathrm{M}$ GTD and absence of GTD. The dissociation rates of mant-ADP from 6IQ were measured using fluorescence energy transfer between the tryptophans of MD of 6IQ and mant-ADP. The transient shown is the average of six separate recordings. The red line is a single exponential fit yielding a rate constant of $403 \mathrm{~s}^{-1}$ in the absence of GTD (left) and $24 \mathrm{~s}^{-1}$ in the presence of $1 \mu \mathrm{M}$ GTD (right). The average of three independent assays was $22 \pm 3.4 \mathrm{~s}^{-1}$ in the presence of $1 \mu \mathrm{M}$ GTD and $430 \pm 40 \mathrm{~s}^{-1}$ in the absence of GTD. (c) A model of the mechanism by which GTD inhibits the movement of MYA2 in vitro and in vivo. In vitro, in the absence of GTD, the ADP dissociation rate of acto-6IQ is $430 \mathrm{~s}^{-1}$ and the actin sliding velocity of 6IQ is $5.2 \mu \mathrm{m} \mathrm{s}^{-1}$. In the presence of $1 \mu \mathrm{M}$ GTD, the ADP dissociation rate of acto-6IQ is $22 \mathrm{~s}^{-1}$ and the actin sliding velocity of 6IQ is $2.4 \mu \mathrm{m} \mathrm{s}^{-1}$. In vivo, when GTD is not bound to the organelle, it interacts with MD and inhibits ADP dissociation from MD, thus slowing down the velocity of MYA2. In contrast, GTD is bound to the organelle, it does not interact with MD and thus does not inhibit MYA2 movement.

This result shows that $\mathrm{Ca}^{2+}$ did not release the GTD-induced inhibitory effect of MYA2, similar to the results of actin-activated ATPase activities as shown above.

Processivity and stepping dynamics of MYA2. Next, we investigated the processivity of single MYA2 Full using TIRFM. Although MYA2 fused with GFP at the MD showed non-processive motion along an actin filament at a saturating concentration of ATP ( $1 \mathrm{mM} \mathrm{ATP})$, it processively moved in one direction at a concentration of $1 \mu \mathrm{M}$ ATP. The fluorescence of GFP showing processive motions photobleached in a single- or doublestep, strongly suggesting single-molecule processivity (Fig. S3).

Then, to dissect the stepping dynamics during the processive motion, we attached a streptavidin-conjugated fluorescent quantum dot (QD) to a biotinylated Halo-tag domain fused at the N-terminus of MD in a MYA2 dimer. We then tracked the motion with $2 \mathrm{~nm}$ precision and $33 \mathrm{~ms}$ time resolution using the FIONA method ${ }^{30}$ (Fig. 4a). We have previously measured the step sizes of myosin Va, which is known to move by a hand-over-hand mechanism, using the same method in this study and confirmed the step size distribution fit to a single Gaussian function with peak at $75 \pm 9 \mathrm{~nm}$ (mean \pm SD) (unpublished result). However, MYA2 showed broader forward step size distribution (Fig. 4b) similar to that of myosin VI which has large and small forward step sizes ${ }^{31,32}$. Therefore, we fit the step size distribution to a three Gaussian function with peaks at $60 \mathrm{~nm}$ (large forward steps), $29 \mathrm{~nm}$ (small forward steps) and $-28 \mathrm{~nm}$ (backward steps). To exclude the possibility that steric hindrance by the QD was responsible for the stepping dynamics, we also examined tetramethyl rhodamine (TMR)-labeled 
a

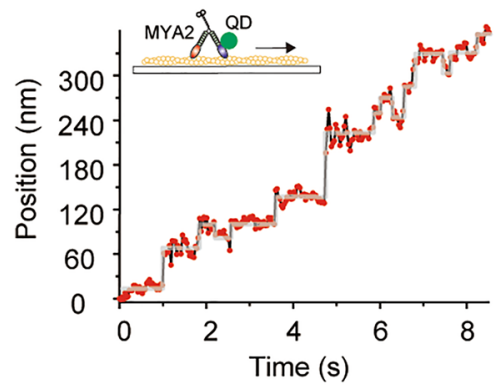

b

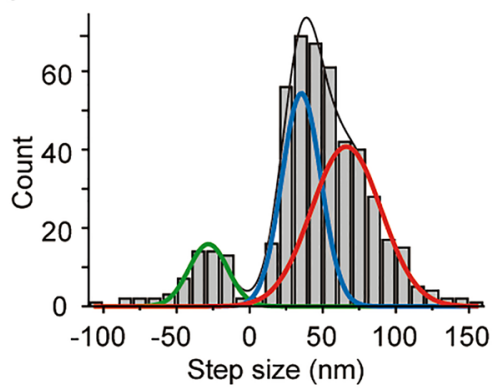

C
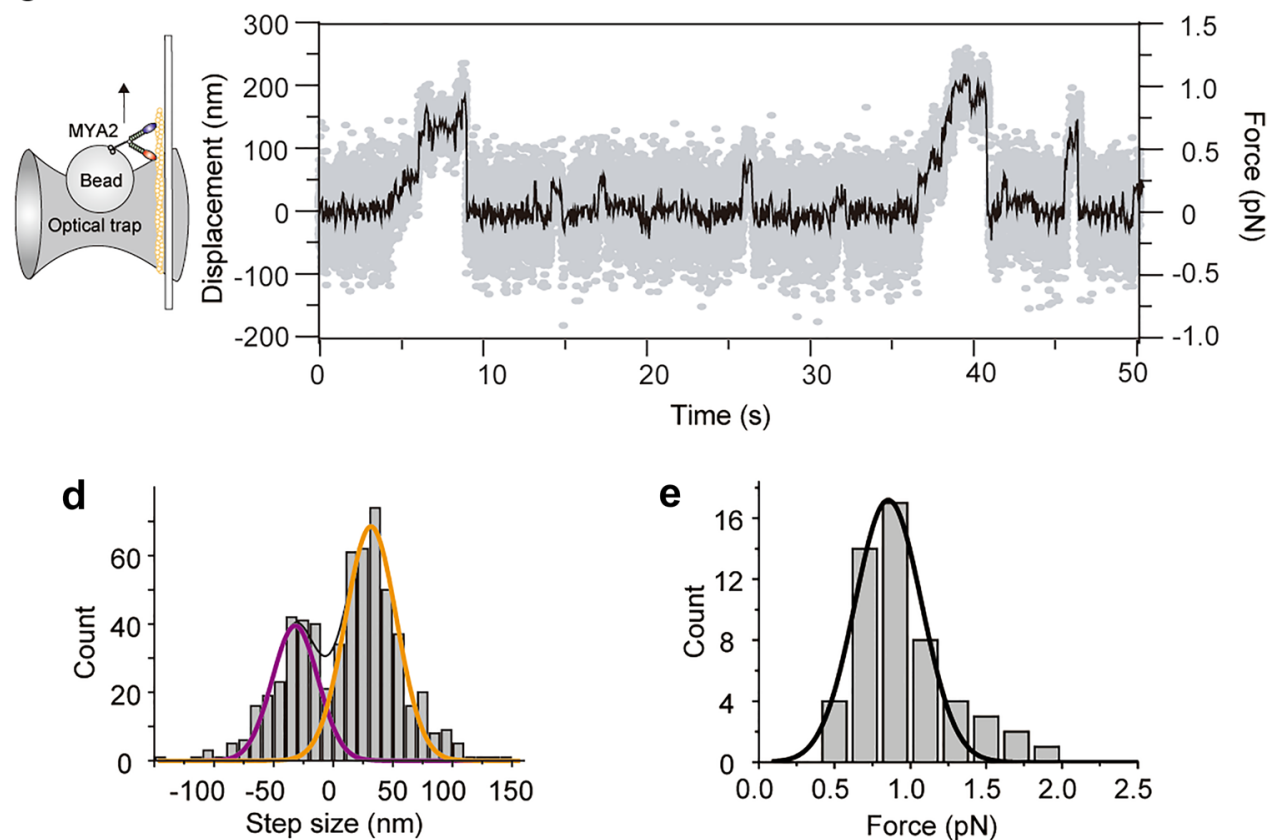

Figure 4. Stepping dynamics and force measurement of MYA2. (a) QD attached to a motor domain in a MYA2 dimer was observed using TIRFM at $2 \mathrm{~nm}$ and $33 \mathrm{~ms}$ spatiotemporal resolution. Steps were analyzed by an automated step-finding algorithm ${ }^{43}$ (gray line). ATP concentration, $1 \mu \mathrm{M}$. (b) Step size histogram of the motor domain. The histogram fit to three Gaussian functions with peaks at $60.5 \pm 14.8 \mathrm{~nm}$ (red line), $29.3 \pm 6.9 \mathrm{~nm}$ (blue line), and $-28.1 \pm 9.9 \mathrm{~nm}$ (green line), respectively. Black line indicates the convolution of three Gaussian functions. (c) Typical trace of optical trapping nanometry at $1 \mu \mathrm{M}$ ATP. Gray line: raw trace; Black line: data passed through a $200 \mathrm{~Hz}$ low pass filter. (d) Step size histogram of an optically trapped bead attached to the MYA2 C-terminal tail. The histogram was fit to two Gaussian functions with peaks at $31.6 \pm 15.2 \mathrm{~nm}$ (orange line), and $-31.9 \pm 13.6 \mathrm{~nm}$ (purple line), respectively. Black line indicates the convolution of the two Gaussian functions. (e) Histogram of MYA2 stall force. The histogram was fitted to single Gaussian function with peaks at $0.85 \pm 0.16 \mathrm{pN}$ (black line).

MYA2. The TMR was labelled at the same position as the QD (a Halo-tag domain fused at the N-terminus of MD). The velocity and run length of TMR-labeled MYA2 were similar to those for QD-labeled MYA2 (Table S1), and the three types of steps were also resolved for TMR-labeled MYA2 (Fig. S4). These results indicated that steric hindrance due to QD labeling at the MD was not significant. A histogram of the dwell time just before the forward steps was fit to a convolution of two exponentials ( $\left.t k^{2} \exp [-k \mathrm{t}]\right)$, in which $k$ is a rate constant (Fig. S5). This is reasonable if we assume that QD-labeled and -nonlabelled MDs alternate steps, each corresponding to the same rate constant, $k^{30}$.

Three types of the step sizes (large and small forward steps, and backward steps) and the dwell time distribution just before the forward steps were consistent with those of myosin VI. In myosin VI, large and small forward steps can be explained by a hand-over-hand motion and inchworm-like motion, respectively ${ }^{31}$. Therefore, we concluded the large $(60 \mathrm{~nm})$ and small $(29 \mathrm{~nm})$ forward steps observed in MYA2 were caused by a hand-overhand and inchworm-like motion, respectively (Fig. 5).

Weak force generation of MYA2. To examine the force generation of MYA2, we attached a fluorescent polystyrene bead to the C-terminal tail and applied force using an optical trap. Figure 4c shows the typical 

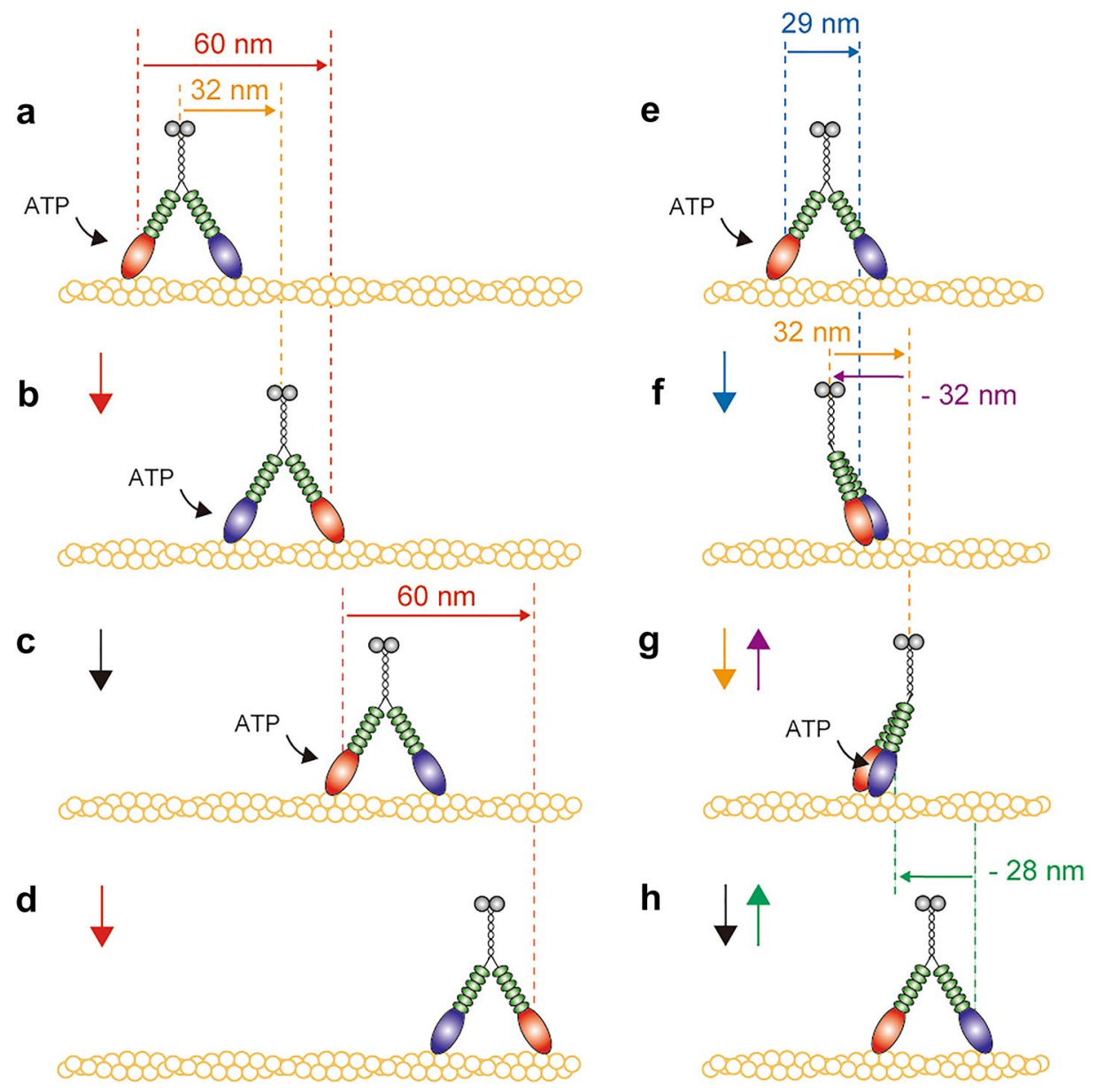

Figure 5. Walking model of MYA2. Left (a-d), large hand-over-hand steps; right (e-h), small inchworm-like steps. Colors denote the following: N-terminal motor domain (red or blue), lever arm (six calmodulins, green), coiled-coil domain (black), and the globular C-terminal tail domain (gray). Red, blue, and green arrows indicate the step of the MD for large, small, and backward steps, respectively, observed using FIONA. Orange and purple arrows indicate the step of the C-terminal tail for forward and backward steps, respectively, observed by optical trapping assay.

trace of a single MYA2 Full under load at $1 \mu \mathrm{M}$ ATP, and the step size histogram is shown in Fig. 4d. We used single-trap geometry and observed the bead displacement, which reported us the C-terminal tail displacements. The histogram was fit to two Gaussian functions with peaks at $32 \mathrm{~nm}$ (forward steps), and - $32 \mathrm{~nm}$ (backward steps). Figure $4 \mathrm{e}$ shows the histogram of the stall force fit to a Gaussian function with peak at $0.85 \pm 0.16 \mathrm{pN}$ $($ mean $\pm S D)$.

\section{Discussion}

In this study, we analyzed the in vitro enzymatic properties of MYA2, which is known to be a major force generator of cytoplasmic streaming in Arabidopsis ${ }^{9}$. The $V_{\max }$ value of the actin-activated ATPase activities measured using recombinant HMM and 6IQ, and the MD of MYA2 were higher than that of myosin $\mathrm{V}$, and similar to that of tobacco $175 \mathrm{kDa}$ myosin XI (Fig. 2a). This high ATPase activity is consistent with MYA2 acting as a driving force for cytoplasmic streaming. In contrast, the $V_{\max }$ value of the ATPase activity of MYA2 Full was much lower than those of HMM, 6IQ, and MD (Fig. 2a). This result indicated that the activity of MYA2 was inhibited by the GTD. Exogenous addition of the GTD inhibited the actin-activated ATPase activities of HMM and 6IQ in a concentration-dependent manner, but not that of $\mathrm{MD}$, suggesting that GTD inhibits ATPase activity via the IQ motif (Fig. 2b). Such GTD suppression was not observed in full-length tobacco $175 \mathrm{kDa}$ myosin XI purified from tobacco BY-2 cells ${ }^{11}$. Autoinhibition using GTD has been reported in myosin V, myosin VIIA, and myosin $\mathrm{X}$. This intramolecular regulation could avoid unproductive movements on actin filaments without cargo and wasteful ATP consumption. The same intramolecular regulation in MYA2 suggests that MYA2 is not merely responsible for driving continuous cytoplasmic streaming, but also for more complex transport equipped with the regulatory system. The result that free GTD slows down ADP dissociation from acto-6IQ (Fig. 3b) indicates that MYA2 stays on actin filaments for a long time until the GTD binds to the cargo. This regulation may contribute to efficient intracellular transport, as in the following hypothesis. When MYA2 is not bound to cargo, it would 
pause on the actin filament while suppressing ATP consumption (Fig. 3c; slow movement in vivo). As soon as the GTD binds to a cargo, MYA2 can participate in the transport (Fig. S6). It has been shown that $\mathrm{Ca}^{2+}$ releases the head-tail interaction and the autoinhibition of myosin $\mathrm{Va}^{23,33}$, and that calmodulin bound to the first IQ motif of myosin $\mathrm{Va}$ is involved in head-tail interaction ${ }^{34}$. In contrast, $\mathrm{Ca}^{2+}$ did not release the autoinhibition of MYA2 (Fig. S2), suggesting that $\mathrm{Ca}^{2+}$ may not be related to the regulation of MYA2, unlike myosin V. We confirmed that calmodulin was bound to the IQ motifs of the purified 6IQ (Fig. S1). However, it was impossible to quantify the molar ratio of calmodulin bound to MYA2 because the amount of purified 6IQ was small and the molecular weight of calmodulin is much smaller than 6IQ, leading to the thin calmodulin band.

The duty ratio of myosin is defined as the ratio of time spent strongly bound to actin to the cycle time of actinactivated ATPase. The time spent strongly bound to actin is the sum of the acto-myosin-ADP (AM.ADP) and acto-myosin $(\mathrm{AM})$ states $^{35}$. Because the AM state is very short for most myosins, the time spent strongly bound to actin is almost equal to that spent in the AM.ADP state, which is approximated by the reciprocal of the ADP dissociation rate from acto-6IQ $\left(1 / 403 \mathrm{~s}^{-1}=2.48 \mathrm{~ms}\right)$. The cycle time of actin-activated ATPase is approximated by the reciprocal of the $V_{\max }$ value of $6 \mathrm{IQ}$ and is $7.87 \mathrm{~ms}$. Thus, the duty ratio of MYA2 is expected to be $32 \%$ $(100 \times 2.48 \mathrm{~ms} / 7.87 \mathrm{~ms}=32 \%)$, showing that MYA2 is a non-processive myosin.

The single-molecule motility of MYA2 revealed its unique motility, which is quite different from that previously reported for tobacco $175 \mathrm{kDa}$ mosin $\mathrm{XI}^{11}$. Optical nanometry of single MYA2 molecules using TIRFM revealed that MYA2 was non-processive at high ATP concentrations, which is consistent with the duty ratio estimation. However, MYA2 demonstrated processive motion on an actin filament at low ATP concentrations. FIONA revealed that one MD in a MYA2 dimer moved with three different step sizes: $-28 \mathrm{~nm}, 29 \mathrm{~nm}$, and $60 \mathrm{~nm}$, and an optical trapping assay revealed the C-terminal tail moved with $\pm 32 \mathrm{~nm}$ step sizes. Based on this observation, we propose the model for MYA2 step movement shown in Fig. 5. Forward large steps $(60 \mathrm{~nm})$ are explained by a hand-over-hand mechanism in Fig. 5a-d. First, the trailing MD unbinds from actin upon ATP binding, moves forward and binds to the forward actin target $60 \mathrm{~nm}$ ahead (Fig. 5a,b). Second, the new trailing MD moves forward in the same manner (Fig. 5b-d). Because the QD was labeled in one MD of a dimer, two ATPase cycles were required before our observation of the $60 \mathrm{~nm}$ forward steps. This is consistent with the dwell time distribution explained by a convolution of two exponentials (Fig. S5). The hand-over-hand mechanism shows the C-terminal tail moves forward $32 \mathrm{~nm}$ as observed by an optical trapping assay (Fig. $4 \mathrm{~d}$ ). Forward small steps $(29 \mathrm{~nm})$ are shown in Fig. 5e-h by an inchworm-like mechanism. Here, the trailing MD unbinds from actin upon ATP binding and binds to the forward actin target $29 \mathrm{~nm}$ ahead (Fig. 5e,f). Then, the two lever arms tilt and the C-terminal tail moves forward $32 \mathrm{~nm}$ (Fig. 5f,g). Finally, the leading MD undergoes a small forward step (Fig. 5g,h) to return to Fig. 5e. state. For backward steps of $-28 \mathrm{~nm}$ of MD, the reversal motion from Fig. $5 \mathrm{f}$ to e or from Fig. $5 \mathrm{~h}$ to $\mathrm{g}$ is probable. However, the lever arm swing (Fig. $5 \mathrm{f}, \mathrm{g}$ ) should be a fast transition, therefore, the reversal motion from Fig. $5 \mathrm{f}$ to e is not considered in our model. Such a reversal motion is inhibited in myosin VI stepping ${ }^{31}$. For backward steps of $-32 \mathrm{~nm}$ by the C-terminal tail, a reversal motion by the hand-over-hand mechanism is inhibited because $-60 \mathrm{~nm}$ steps of MD were not observed. Instead, the most probable reversal motion of the lever arm swing is an inchworm-like mechanism (from Fig. $5 \mathrm{~g}$ to $\mathrm{f}$ ).

Force measurement using optical trapping showed that the stall force of MYA2 was $0.85 \mathrm{pN}$, which was similar to that of tobacco $175 \mathrm{kDa}$ myosin XI $(0.5 \mathrm{pN})$ and less than half that of myosin $\mathrm{V}(2-3 \mathrm{pN})^{36,37}$. These results from single molecular analysis suggested that MYA2 properly uses dual stepping mode by hand-overhand and inchworm mechanism in vivo. Proper use of dual stepping mode may be advantageous in avoiding interference between myosin molecules when many motors are closely associated on the same organelles. That is, if a certain myosin interferes with the myosin in front of it, it is possible to briefly pause the movement of the trailing myosin by its stepping back a small half step, without large backstepping. MYA2 may be a suitable motor for driving large organelles, such as the ER with multiple molecules simultaneously. Since the organelle carried by MYA2 and the number of MYA2 molecules working on the organelle have not been clarified, further cell biological analysis is required.

Tobacco $175 \mathrm{kDa}$ myosin XI was phylogenetically the closest to MYA2 among the 13 Arabidopsis myosin XI members. However, considerable differences were found in the mode of movement (Table S2) and in their regulation, suggesting that myosin XIs have diverse molecular functions.

\section{Methods}

Protein engineering and expression. A baculovirus transfer vector for pFastBacMYA2 was generated as follows. MYA2 (AGI code: AT5g43900.1, UNIPROT ID: Q9LKB9) cDNA cloned from Arabidopsis seedlings was mutated to create an NcoI site at the upstream region of the nucleotide sequence encoding residue 1 , and an AgeI site in the downstream region of the nucleotide sequence encoding residue 4515 of MYA2. These sequences were cut with NcoI and AgeI and the fragment was ligated with the NcoI-AgeI fragment of the pFastBac MD with a FLAG-tag 27 . The resulting construct, MYA2, encodes the N-terminal sequence (MSYYHHHHHHDYKDDDDKNIPTTENLYFQGA) containing a His ${ }_{6}$-tag and a FLAG-tag (DYKDDDDK), residues 1-1505 of MYA2, a flexible linker (GGG), a Myc-epitope sequence (EQKLISEEDL), and a His for pFastBac Halo-tag-fused MYA2 was generated as follows. The pFN21A (HaloTag (R) 7) CMV Flexi (R) Vector (Promega Corporation, Madison, USA) was mutated to create an NcoI site downstream of the nucleotide sequence encoding the HaloTag 7 open reading frame and was cut with NcoI. The NcoI digestion fragment was ligated with the NcoI digestion fragment of pFastBacMYA2. The resultant construct, Halo-tag-fused MYA2, encodes the N-terminal sequence (MSYYHHHHHHDYKDDDDKNIPTTENLYFQGA) containing a His ${ }_{6}$-tag, a FLAG-tag (DYKDDDDK), and a Halo-tag 7, residues 1-1505 of MYA2, a flexible linker (GGG), a Myc-epitope sequence (EQKLISEEDL), and a His - $_{8}$-tag. A baculovirus transfer vector for pFastBac Arabidopsis calmodulin was generated as follows. Arabidopsis calmodulin (AT3G56800.1) cDNA cloned from Arabidopsis seedlings was 
mutated to create an XbaI site upstream of the nucleotide sequence encoding residue 1 , and an XhoI site downstream of the nucleotide sequence encoding residue 448 of Arabidopsis calmodulin. The XbaI-XhoI digestion fragment was ligated with the XbaI-XhoI digestion fragment of pFastBac 1 (Invitrogen, Carlsbad, CA, USA). To express MYA2 and Halo-tag fused MYA2, $800 \mathrm{~mL}$ of High Five TM cell culture (Invitrogen) was infected with viruses expressing the respective constructs. Arabidopsis calmodulin was co-produced by co-infection with a virus expressing Arabidopsis calmodulin. The infected cells were cultured in Erlenmeyer flasks $(1 \mathrm{~L} \times 4)$ at $28^{\circ} \mathrm{C}$ and shaken at $130 \mathrm{rpm}$, for $43 \mathrm{~h}$. The study complies with national guidelines in Japan.

Protein purification. Protein purification was performed as previously described with some modifications ${ }^{35}$. Cells were harvested and washed with $150 \mathrm{mM} \mathrm{NaCl}, 1 \mathrm{mM}$ EGTA, and $10 \mathrm{mM}$ HEPES, pH 7.4. The pelleted cells were suspended with $2 \mathrm{vol} / \mathrm{g}$ cells of buffer A (30 mM HEPES, pH 7.4, $200 \mathrm{mM} \mathrm{KCl}, 5 \mathrm{mM} \mathrm{MgCl}, 4 \mathrm{mM}$ ATP, $1 \mathrm{mM}$ EGTA, $1 \mathrm{mM}$ DTT, and a mixture of protease inhibitors). Then, $2 \mathrm{vol} / \mathrm{g}$ cells of buffer A containing $2 \%$ Nonidet P-40 was added and mixed. After incubation on ice for $10 \mathrm{~min}$, the lysate was centrifuged at $228,000 \times g$ for $30 \mathrm{~min}$. The supernatant was mixed with $0.3 \mathrm{~mL}$ of anti-FLAG M2 affinity resin (Sigma-Aldrich, St. Louis, MO, USA) in a $50 \mathrm{~mL}$ tube on a rotating wheel for one hour at $4{ }^{\circ} \mathrm{C}$. The resin suspension was then loaded on a column and washed with $30 \mathrm{~mL}$ of buffer A containing $1 \mu \mathrm{M}$ mouse calmodulin. Halo-tag-fused MYA2 was biotinylated by incubation with buffer A containing $10 \mu \mathrm{M}$ of HaloTag PEG-Biotin Ligand (Promega) in the column for $10 \mathrm{~min}$. MYA2 and Halo-tag-fused MYA2 was eluted with buffer A containing $0.2 \mathrm{mg} / \mathrm{mL}$ of $3 \times$ FLAG peptide (Sigma-Aldrich). Arabidopsis calmodulin was expressed in High Five TM cells by infecting them with virus expressing Arabidopsis calmodulin and purified using the method of Awata et al. ${ }^{38}$. Gallus gallus skeletal muscle actin was prepared using method of Spudich and Watt ${ }^{39}$.

ATPase activity. ATPase activities were determined by measuring released phosphate as previously described ${ }^{40}$. The reaction mixtures for the assay of actin-activated $\mathrm{Mg}^{2+}$-ATPase activity contained $25 \mathrm{mM} \mathrm{KCl}$, $4 \mathrm{mM} \mathrm{MgCl}_{2}, 25 \mathrm{mM}$ HEPES-KOH (pH 7.4), $3 \mathrm{mM} \mathrm{ATP,} 1 \mathrm{mM} \mathrm{DTT}, 1 \mathrm{mg} / \mathrm{mL} \mathrm{BSA}$, and, at $25^{\circ} \mathrm{C}, 0.125-4 \mathrm{mg} /$ mL F-actin.

In vitro gliding assay. The velocity was measured using an anti-myc antibody-based version of the in vitro actin filament gliding assay as previously described ${ }^{27}$. The velocity of actin filaments was measured in $150 \mathrm{mM}$ $\mathrm{KCl}, 4 \mathrm{mM} \mathrm{MgCl}$, $25 \mathrm{mM}$ HEPES-KOH (pH 7.4), $2 \mathrm{mM} \mathrm{ATP,} 10 \mathrm{mM}$ DTT, and oxygen scavenger system $\left(120 \mu \mathrm{g} / \mathrm{mL}\right.$ glucose oxidase, $12.8 \mathrm{mM}$ glucose, and $20 \mu \mathrm{g} / \mathrm{mL}$ catalase) at $25^{\circ} \mathrm{C}$. Average sliding velocities were determined by measuring the displacements of actin filaments.

Measurement of ADP dissociation rate using a stopped-flow apparatus. Experiments were done in $25 \mathrm{mM} \mathrm{KCl}, 4 \mathrm{mM} \mathrm{MgCl} 2,1 \mathrm{mM} \mathrm{DTT}$, and $25 \mathrm{mM}$ Hepes pH 7.4, at $25^{\circ} \mathrm{C}$, using an Applied Photophysics SX18MV stopped-flow spectrophotometer (dead time: $1.15 \mathrm{~ms}$ ). The mixture of $0.4 \mu \mathrm{M} 6 \mathrm{IQ}, 60 \mu \mathrm{M}$ mant-ADP, $20 \mu \mathrm{M}$ actin in the presence $(1.6 \mu \mathrm{M})$ or absence of GTD was mixed with $3 \mathrm{mM}$ ATP (each concentration is the concentration after mixing). Mant-ADP were excited at $290 \mathrm{~nm}$ via fluorescence resonance energy transfer from tryptophan of MD, and emission was observed after passing through a $389 \mathrm{~nm}$ cutoff filter. Dissociation of mantADP from was monitored by the decrease of its fluorescence.

Single-molecule imaging. Single-molecule imaging was performed as previously described ${ }^{31}$. Briefly, the Halo-tag domain fused at the N-terminus of MD was attached with HaloTag PEG-Biotin Ligand (G859A, Promega) or HaloTag TMR Ligand (G825A, Promega) during the purification of MYA2. Then, streptavidinconjugated QD (Qdot585, Q10113MP (Invitrogen)) and biotinylated-MYA2 were mixed at a molar ratio of 1:1 and incubated overnight on ice. Assay buffer (AB; $30 \mathrm{mM} \mathrm{HEPES-KOH} \mathrm{[pH} \mathrm{7.8],} 25 \mathrm{mM} \mathrm{KCl,} 5 \mathrm{mM} \mathrm{MgCl}$, and $2 \mathrm{mM}$ EGTA) was prepared before each experiment. Sample chambers were assembled using double-sided transparent tape (Scotch) and dried coverslips. Actin filaments were quickly flowed into sample chambers in which $\alpha$-actinin molecules were directly adhered to a glass slide. The glass surface was then coated with $5 \mathrm{mg} / \mathrm{mL}$ casein. A 1:1 QD-MYA2 mixture was diluted 100 times in motility buffer (MB; AB plus, $1 \mu \mathrm{M}$ ATP, $0.2 \mathrm{mg} / \mathrm{mL}$ glucose oxidase, $4.5 \mathrm{mg} / \mathrm{mL}$ glucose, $36 \mathrm{mg} / \mathrm{mL}$ catalase, $1 \% \mathrm{v} / \mathrm{v} 2$-mercaptoethanol ${ }^{41}, 2 \mathrm{mM}$ phosphocreatine, $0.1 \mathrm{mg} / \mathrm{mL}$ creatine phosphokinase ${ }^{32}, 0.1 \mathrm{mg} / \mathrm{mL}$ calmodulin). This mixture was added into the sample chamber, and the chamber was sealed with nail polish and observed immediately. QD-conjugated MYA2 movement was imaged using TIRFM, and the excitation was provided by a $405 \mathrm{~nm}$ laser light (Compass405-50CW; Coherent, Santa Clara, CA, USA). The fluorescent photons were collected with a back-illuminated EMCCD camera (DV887ECS-BV; Andor Technology, Belfast, Northern Ireland). The fluorescence was passed through a dichroic mirror (DML557 nm; Asahi Spectra, Tokyo, Japan) and emission filter (FF01-593/40-25, Semrock, Rochester, NY, USA). Image acquisition was performed by commercial software (Andor, SOLIS software). The sample was kept at ambient temperature during data collection $\left(27 \pm 2{ }^{\circ} \mathrm{C}\right)$. Exported 8-bit data was analyzed by a program custom written in LabVIEW (National Instruments, Austin, TX, USA). The spot center for each frame was determined using a two-dimensional Gaussian fit according to a published method ${ }^{30,42}$. The accuracy of detection of the spot center was $2.0 \mathrm{~nm}$. All steps were analyzed by an automated step-finding algorithm ${ }^{43}$.

Optical trapping. Optical trapping was performed as previously described, with some modifications ${ }^{44}$. Briefly, carboxylate modified polystyrene beads (Invitrogen; $0.2 \mu \mathrm{m}$ in diameter) were cross-linked to c-Myc monoclonal antibody (Clontech, Shiga, Japan) and coated with $10 \mathrm{mg} / \mathrm{mL}$ BSA. One microliter of the beads and c-myc tagged MYA2 were mixed and incubated for $30 \mathrm{~min}$ in AB containing $10 \mathrm{mg} / \mathrm{mL}$ BSA at a ratio of 1:20. 
The ratio was adjusted so that less than $10 \%$ of the beads displayed movement. Sample chambers were assembled using double-sided transparent tape (Scotch) and dried coverslips. Fluorescent dye-labeled actin filaments were quickly flowed into sample chambers in which $\alpha$-actinin molecules were directly adhered to a glass slide. The glass surface was then coated with $5 \mathrm{mg} / \mathrm{mL}$ casein. One microliter of MYA2 coated beads was diluted 100 times in MB. This mixture was added to the sample chamber, and the chamber was sealed with nail polish and observed immediately. The optics of the optical trapping and detection of the bead positions were conducted as previously described ${ }^{32,44}$. Bead displacements were recorded at a sampling rate of $24 \mathrm{kHz}$ with a bandwidth of $10 \mathrm{kHz}$. The load exerted on the beads was calculated from the bead displacement multiplied by the trap stiffness $(\sim 7.4 \mathrm{fN} / \mathrm{nm})$, which was determined from the variance of the Brownian motion of a trapped bead by the equipartition theorem of energy. We determined the step sizes after passing the data through a low pass filter with a bandwidth of $200 \mathrm{~Hz}$, using a custom program in DADiSP (CAE Solutions, Burlington, MA, USA) and Visual C++(Microsoft, Redmond, WA, USA). All steps were checked by eye. To determine the precise step size at low loads, we calculated the elastic component in our single trapping geometry and estimated the correction factor depending on load ${ }^{45}$.

Received: 30 August 2021; Accepted: 8 February 2022

Published online: 24 February 2022

\section{References}

1. Kollmar, M. \& Mühlhausen, S. Myosin repertoire expansion coincides with eukaryotic diversification in the Mesoproterozoic era. BMC Evol. Biol. 17, 211-212 (2017).

2. Sweeney, H. L. \& Holzbaur, E. L. F. Motor proteins. Cold Spring Harb. Perspect. Biol. 10, a021931. https://doi.org/10.1101/cshpe rspect.a021931 (2018).

3. Titus, M. A. Myosin-driven intracellular transport. Cold Spring Harb. Perspect. Biol. 10, a021972. https://doi.org/10.1101/cshpe rspect.a021972 (2018).

4. Hartman, M. A., Finan, D., Sivaramakrishnan, S. \& Spudich, J. A. Principles of unconventional myosin function and targeting. Annu. Rev. Cell Dev. Biol. 27, 133-155 (2011).

5. Heissler, S. M. \& Sellers, J. R. Kinetic adaptations of myosins for their diverse cellular functions. Traffic 17, 839-859 (2016).

6. Liu, J., Taylor, D. W., Krementsova, E. B., Trybus, K. M. \& Taylor, K. A. Three-dimensional structure of the myosin V inhibited state by cryoelectron tomography. Nature 442, 208-211 (2006).

7. Li, J. F. \& Nebenführ, A. The tail that wags the dog: The globular tail domain defines the function of myosin V/XI. Traffic 9, 290-298 (2008).

8. Nascimento, A. F. Z. et al. Structural insights into functional overlapping and differentiation among myosin V motors. J. Biol. Chem. 288, 34131-34145 (2013).

9. Tominaga, M. \& Ito, K. The molecular mechanism and physiological role of cytoplasmic streaming. Curr. Opin. Plant Biol. 27, 104-110(2015).

10. Shimmen, T. The sliding theory of cytoplasmic streaming: Fifty years of progress. J. Plant Res. 120, 31-43 (2007).

11. Tominaga, M. et al. Higher plant myosin XI moves processively on actin with $35 \mathrm{~nm}$ steps at high velocity. EMBO J. 22, $1263-1272$ (2003).

12. Reddy, A. S. \& Day, I. S. Analysis of the myosins encoded in the recently completed Arabidopsis thaliana genome sequence. Genome Biol. 2, 1-19 (2001) (Epub 2001 Jul 3).

13. Peremyslov, V. V., Klocko, A. L., Fowler, J. E. \& Dolja, V. V. Arabidopsis myosin XI-K localizes to the motile endomembrane vesicles associated with F-actin. Front. Plant. Sci. 3, 184 (2012).

14. Peremyslov, V. V., Prokhnevsky, A. I., Avisar, D. \& Dolja, V. V. Two class XI myosins function in organelle trafficking and root hair development in Arabidopsis. Plant Physiol. 146, 1109-1116 (2008).

15. Peremyslov, V. V., Prokhnevsky, A. I. \& Dolja, V. V. Class XI myosins are required for development, cell expansion, and F-Actin organization in Arabidopsis. Plant Cell 22, 1883-1897 (2010).

16. Prokhnevsky, A. I., Peremyslov, V. V. \& Dolja, V. V. Overlapping functions of the four class XI myosins in Arabidopsis growth, root hair elongation, and organelle motility. Proc. Natl. Acad. Sci. USA. 105, 19744-19749 (2008).

17. Ueda, H. et al. Myosin-dependent endoplasmic reticulum motility and F-actin organization in plant cells. Proc. Natl. Acad. Sci. USA 107, 6894-6899 (2010).

18. Ojangu, E. et al. Myosins XI-K, XI-1, and XI-2 are required for development of pavement cells, trichomes, and stigmatic papillae in Arabidopsis. BMC Plant Biol. 12, 81 (2012).

19. Haraguchi, T. et al. Functional diversity of class XI myosins in Arabidopsis thaliana. Plant Cell Physiol. 59, 2268-2277 (2018).

20. Tominaga, M. \& Nakano, A. Plant-specific myosin XI, a molecular perspective. Front. Plant Sci. 3, 211 (2012).

21. Haraguchi, T., Tominaga, M., Nakano, A., Yamamoto, K. \& Ito, K. Myosin XI-I is mechanically and enzymatically unique among class-XI myosins in Arabidopsis. Plant Cell Physiol. 57, 1732-1743 (2016).

22. Thirumurugan, K., Sakamoto, T., Hammer, J. A. 3rd., Sellers, J. R. \& Knight, P. J. The cargo-binding domain regulates structure and activity of myosin 5 . Nature 442, 212-215 (2006).

23. Wang, F. et al. Regulated conformation of myosin V. J. Biol. Chem. 279, 2333-2336 (2004).

24. De La Cruz, E. M., Wells, A. L., Rosenfeld, S. S., Ostap, E. M. \& Sweeney, H. L. The kinetic mechanism of myosin V. Proc. Natl. Acad. Sci. USA. 96, 13726-13731 (1999).

25. De La Cruz, E. M., Ostap, E. M. \& Sweeney, H. L. Kinetic mechanism and regulation of myosin VI. J. Biol. Chem. 276, 32373-32381 (2001).

26. Trybus, K. M., Krementsova, E. \& Freyzon, Y. Kinetic characterization of a monomeric unconventional myosin V construct. J. Biol. Chem. 274, 27448-27456 (1999).

27. Ito, K. et al. Kinetic mechanism of the fastest motor protein, Chara myosin. J. Biol. Chem. 282, 19534-19545 (2007).

28. Kambara, T., Komaba, S. \& Ikebe, M. Human myosin III is a motor having an extremely high affinity for actin. J. Biol. Chem. 281, 37291-37301 (2006).

29. Haraguchi, T. et al. Molecular characterization and subcellular localization of Arabidopsis class VIII myosin, ATM1. J. Biol. Chem. 289, 12343-12355 (2014).

30. Yildiz, A. et al. Myosin V walks hand-over-hand: Single fluorophore imaging with 1.5-nm localization. Science 300, 2061-2065 (2003).

31. Nishikawa, S. et al. Switch between large hand-over-hand and small inchworm-like steps in myosin VI. Cell 142, 879-888 (2010). 
32. Iwaki, M., Iwane, A. H., Shimokawa, T., Cooke, R. \& Yanagida, T. Brownian search-and-catch mechanism for myosin-VI steps. Nat. Chem. Biol. 5, 403-405 (2009).

33. Sellers, J. R., Thirumurugan, K., Sakamoto, T., Hammer, J. A. 3rd. \& Knight, P. J. Calcium and cargoes as regulators of myosin 5a activity. Biochem. Biophys. Res. Commun. 369, 176-181 (2008).

34. Lu, Z. et al. Calmodulin bound to the first IQ motif is responsible for calcium-dependent regulation of myosin 5a. J. Biol. Chem. 287, 16530-16540 (2012).

35. Ito, K., Yamaguchi, Y., Yanase, K., Ichikawa, Y. \& Yamamoto, K. Unique charge distribution in surface loops confers high velocity on the fast motor protein Chara myosin. Proc. Natl. Acad. Sci. USA. 106, 21585-21590 (2009)

36. Mehta, A. D. et al. Myosin-V is a processive actin-based motor. Nature 400, 590-593 (1999).

37. Iwaki, M., Iwane, A. H., Ikezaki, K. \& Yanagida, T. Local heat activation of single myosins based on optical trapping of gold nanoparticles. Nano Lett. 15, 2456-2461 (2015).

38. Awata, J., Saitoh, K., Shimada, K., Kashiyama, T. \& Yamamoto, K. Effects of $\mathrm{Ca}^{2+}$ and calmodulin on the motile activity of characean myosin in vitro. Plant Cell Physiol. 42, 828-834 (2001).

39. Spudich, J. A. \& Watt, S. The regulation of rabbit skeletal muscle contraction. I. Biochemical studies of the interaction of the tropomyosin-troponin complex with actin and the proteolytic fragments of myosin. J. Biol. Chem. 246, 4866-4871 (1971).

40. Ito, K. et al. Recombinant motor domain constructs of Chara corallina myosin display fast motility and high ATPase activity. Biochem. Biophys. Res. Commun. 312, 958-964 (2003).

41. Harada, Y., Sakurada, K., Aoki, T., Thomas, D. D. \& Yanagida, T. Mechanochemical coupling in actomyosin energy transduction studied by in vitro movement assay. J. Mol. Biol. 216, 49-68 (1990).

42. Thompson, R. E., Larson, D. R. \& Webb, W. W. Precise nanometer localization analysis for individual fluorescent probes. Biophys. J. 82, 2775-2783 (2002).

43. Kerssemakers, J. W. et al. Assembly dynamics of microtubules at molecular resolution. Nature 442, 709-712 (2006).

44. Iwaki, M. et al. Cargo-binding makes a wild-type single-headed myosin-VI move processively. Biophys. J. 90, 3643-3652 (2006).

45. Kojima, H., Muto, E., Higuchi, H. \& Yanagida, T. Mechanics of single kinesin molecules measured by optical trapping nanometry. Biophys. J. 73, 2012-2022 (1997).

\section{Acknowledgements}

This work was supported by JSPS KAKENHI (no. 920001009 , no. 23770060 , and no. 25221103 to M.T.) (no. JP 20K06583, no. JP 17K07436, and 15H01309 to K.I.) (no. 21710120 and no. 26440131 to M.I.), and from the Japan Science and Technology Agency, ALCA, JPMJAL1401 (to M.T. and K.I.).

\section{Author contributions}

M.T., K.I, and M.I. conceived the idea and design the study. T.H., T.M., N.S., K.Y., and A.K. conducted the experiments, T.H., I.K., M.I., K.Y. and A.K. analyzed the results. M.T., K.I, and M.I. contributed to the writing of the manuscript. All authors reviewed the manuscript.

\section{Competing interests}

The authors declare no competing interests.

\section{Additional information}

Supplementary Information The online version contains supplementary material available at https://doi.org/ 10.1038/s41598-022-07047-0.

Correspondence and requests for materials should be addressed to K.I., M.I. or M.T.

Reprints and permissions information is available at www.nature.com/reprints.

Publisher's note Springer Nature remains neutral with regard to jurisdictional claims in published maps and institutional affiliations.

(c) (i) Open Access This article is licensed under a Creative Commons Attribution 4.0 International License, which permits use, sharing, adaptation, distribution and reproduction in any medium or format, as long as you give appropriate credit to the original author(s) and the source, provide a link to the Creative Commons licence, and indicate if changes were made. The images or other third party material in this article are included in the article's Creative Commons licence, unless indicated otherwise in a credit line to the material. If material is not included in the article's Creative Commons licence and your intended use is not permitted by statutory regulation or exceeds the permitted use, you will need to obtain permission directly from the copyright holder. To view a copy of this licence, visit http://creativecommons.org/licenses/by/4.0/.

(c) The Author(s) 2022 